\title{
Nesting in perception of affordances for stepping and leaping
}

\author{
Jeffrey B. Wagman ${ }^{1}$ • Jiuyang Bai ${ }^{1}$ • Peter J. K. Smith ${ }^{2}$
}

Published online: 24 May 2016

(C) The Psychonomic Society, Inc. 2016

\begin{abstract}
Perception of affordances for a given behavior typically reflects the task-specific action capabilities of the perceiver. However, many experiments have shown a discrepancy between the perceptual and behavioral boundaries for a given behavior. One possibility for such a discrepancy is that the context of many experimental tasks transformed what is typically a dynamic perception-action task into an analytical or reflective judgment. We investigated this hypothesis with respect to perception of maximum stepping and leaping distance. For both behaviors, perception of these affordances more closely reflected action capabilities when the perceptual task was nested within a superordinate task than when it was not (regardless of whether the behavior itself was performed). Additionally, verbal reports of perception of maximum leaping distance more closely reflected action capabilities when there was an explicit time limit on such reports. The results are discussed in the context of the ecological principle of nesting and in attentional focus during motor control tasks.
\end{abstract}

Keywords Perception and Action · Embodied perception . Motor control

An affordance is an emergent opportunity for performing a particular behavior given the fit between action capabilities and environmental properties. Decades of research on

Jeffrey B. Wagman

jeffreywagman@illinoisstate.edu

1 Department of Psychology, Illinois State University, Campus Box 4620, Normal, IL 61790-4620, USA

2 School of Kinesiology and Recreation, Illinois State University, Normal, IL, USA perception of affordances has shown that perception of whether a particular behavior can be performed generally reflects the dynamic and task-specific action capabilities of the perceiver. That is, such research has shown that perception of affordances is action-scaled. For example, researchers have found that perception of whether a surface could be stood on depended on the length, strength, and flexbility of the legs (Konczak, Meeuwsen, \& Cress 1992; Warren, 1984), perception of whether an object could be reached reflected (impending changes to) reaching ability (Carello, Grosofsky, Reichel, Solomon, \& Turvey, 1989; Wagman \& Morgan, 2010), and perception of whether a surface could be stood on reflected how a weighted backpack changed the person's center of mass (Regia-Corte \& Wagman, 2008).

Despite such action scaling of perception of affordances, many such experiments have shown a discrepancy between the perceptual boundary for a given behavior and the behavioral boundary on that behavior. That is, such experiments have shown that perception of affordances was not perfectly action scaled. For example, in such experiments, people have generally underestimated maximum reaching height (Wagman \& Morgan, 2010), maximum leaping distance (Cole, Chan, Vereijken, \& Adolph, 2013; Day, Wagman \& Smith, 2015), and their ability to stand on an inclined surface while wearing a weighted backpack (Regia-Corte \& Wagman, 2008).

There are a number of possible explanations for such discrepancies, some of which are more theoretically satisfying than others. First, underestimations of action capabilities could be interpreted as a (conscious or unconscious) "safety margin" on the part of the perceiver to avoid collisions, falls, injuries, or other accidents. While this is possible in at least some cases, it does not explain cases in which participants overestimate their action capabilties. For example, research has shown that novice wheelchair users generally overestimated their ability to roll through an aperture 
(Higuchi, Takada, Matsuura, \& Imanaka, 2004), and toddlers overestimated their ability to walk down slopes (Adolph, 2008). It is also does not explain cases in which perceivers underestimate their ability to perform a behavior that poses little or no accident or injury risk (e.g., overhead reaching while standing on the floor; Wagman \& Morgan, 2010).

Second, such discrepancies could be interpreted as (1) insufficient attunement to a (or the) stimulation pattern that is informative about affordaces for that behavior and/or (2) insufficient calibration to the fit between a a particular action capability and a particular environmental property. Such an explanation is certainly plausible given that experimental findings that have shown that repeated experience perceiving affordances for a given behavior (or repeated practice performing the behavior itself) can be sufficient to bring perceptual boundaries for that behavior into closer correspondence with the behavioral boundary (e.g., Franchak \& Adolph, 2014; Franchak, ver der Zalm, \& Adolph, 2010; Mark, 1987; Stoffregen, Yang, Giveans, Flanagan, \& Bardy, 2009; Yasuda, Wagman, \& Higuchi, 2014).

Third, such discrepancies could be due to the artificiality of some affordance perception tasks. For example, in many experiments investigating perception of affordances, participants have been asked to report on the absolute limits of their ability to perform a given behavior (e.g., "what is your maximum reaching height?") rather than on their preferred means of performing that behavior (e.g., "would you choose to reach to this height?"). Although participants may be capable of performing a behavior at the limits of their ability, doing so may be awkward, uncomfortable, or even dangerous. As a result, they may not choose to do so, especially if another means of performing the same behavior is possible. For example, Mark, Nemeth, Gardner, Dainoff, Paasche, Duffy, \& Grandt (1997) found that the perception of maximum reaching distance in an arm-only reaching task differed from the distance at which participants spontaneously transitioned from an arm-only reach to an arm-plus-torso reach. Similarly, Wagman $\&$ Malek (2009) found differences in willingness to perform a given behavior across conditions that did not produce differences in perceptual boundaries for that behavior.

Similarly, in many experiments on perception of affordances, participants have been asked to make explicit verbal reports about whether or not a particular behavior could be performed and have been given an unlimited amount of time in which to do so. In everyday settings, of course, perception of affordances for a given behavior is implicitly expressed through performance of that behavior (or by a decision not to perform the behavior). Moreover, in many everyday settings, such a decision must be made in the limited time span over which that behavior is possible. Consequently, the context of many experimental tasks may have transformed what is typically a dynamic perception-action task into an analytical or reflective judgment (Heft, 1993). In particular, isolating the perceptual task from the larger context in which it occurs may have contributed to the differences between perceptual and behavioral boundaries observed in many experiments.

Accordingly, Heft (1993) found that perception of whether an object was within reach was better calibrated to actual maximum reaching distance when either (1) the perceptual task was nested within the context of performing the behavior (i.e., participants chose whether or not to grasp a pen at a given distance for use in a drawing task) or (2) there was an explicit time limit on making a verbal perceptual report than when (3) the perceptual task was the primary (or focal) task and there was no explicit time limit. In other words, Heft (1993) found that perception of affordances for reaching more closely corresponded to actual reaching ability when the context of the experimental task preserved (at least some of) the larger context in which the perceptual task typically ocurrs. We attempted to generalize these findings to perception of affordances for two other behaviors that are at least to some extent, the lower body equivalents of reaching - stepping or leaping over a gap in the support surface. Comparison of perception of affordances for these two behaviors may be instructive given differences in the factors that constrain performance of these behaviors. Maximum stepping distance is primarily constrained by a static geometric property (i.e., leg length). By contrast, maximum distance leaping is a more biomechanically complex behavior that is additionally constrained by dynamic capabilities (e.g., lower body strength, flexibility, and balance). Consequently, perceiving maximum leaping distance may be a more complex task than perceiving maximum stepping distance. Accordingly, perceivers tend to exhibit differences in their ability to perceive affordances for these respective behaviors (Cole et al. 2013; Day et al., 2015). Investigating the effects of context of experimental task on perception of affordances for both such behaviors may provide both stronger and more general support for Heft's conclusions.

Following Heft (1993), we had three specific hypotheses with respect to the effects of context on perception of affordances for each of these behaviors. First, we expected that perception of maximum stepping and leaping distance would be more accurate (i.e., would more closely reflect actual maximum stepping or leaping distance, respectively) when the perceptual task was nested within a superordinate task than when it was not. Second, we expected this to occur both when the superordinate task included performance of a stepping or leaping task and when it did not (but we did not have any specific predictions about the relative accuracy of these two conditions). Third, we expected that verbal reports of perceived maximum stepping or leaping distance would be more accurate when there was an explicit time constraint on making such reports than when there was not. 


\section{Method}

\section{Participants}

One hundred and fifty-nine students (19 men and 140 women) from Illinois State University participated in this experiment. In the interest of participant safety, participants were required to be no heavier than $97.5 \mathrm{~kg}$ (approximately $215 \mathrm{lbs}$.). To avoid a ceiling effect in reports of perceived maximum stepping distance, participants were also required to be no taller than $173 \mathrm{~cm}$ (approximately $5 \mathrm{ft} 8 \mathrm{in}$.). All participants received extra credit in their psychology courses in exchange for their participation. The first 78 participants were assigned to the Stepping Behavior condition, and the next 81 participants were assigned to the Leaping Behavior condition. Within each of these tasks, participants were randomly assigned to one of four Context conditions (Focal, Timed, Puzzle, or Puzzle-Action, see below). One participant was excluded from data analysis due to failure to follow or understand the instructions. The protocol used in this experiment was approved by the Institutional Review Board at Illinois State University in accordance with the Declaration of Helsinki.

\section{Materials and apparatus}

A thin black foam rubber mat (approximately $900 \mathrm{~cm}$ long $\times$ $60 \mathrm{~cm}$ wide) was used for participant safety. A yellow tape mark indicated where each participant was to stand at the beginning of every trial. A thin wooden dowel was placed across the mat at various distances. In the Puzzle and Puzzle-Action conditions, a "connect-the-dots" puzzle was posted on a large moveable whiteboard. A digital scale was used to measure body weight. Tape measures were used to measure maximum stepping distance, maximum leaping distance, and participant standing height. Portions of the Lateral Preference Inventory (Coren, 1993) were used to measure footedness.

\section{Procedure}

Stepping behavior condition Each participant was administered the footedness portion of the Lateral Preference Inventory (Coren, 1993) to determine his/her preferred foot. The experimenter then measured each participant's maximum stepping distance. The participant walked to the other end of the rubber mat and stood with his/her toes just behind a tape mark. He/she was instructed to step as far as possible with his/ her preferred foot as the lead foot and the non-preferred foot as the trail foot. The participant was instructed that during the step, the trail foot must not leave the ground before the lead foot lands and that he/she must (eventually) be able to bring the trail foot (and the rest of the body) in line with the lead foot. The participant paused before bringing the trail foot in line with the lead foot so that the experimenter could measure the distance between the tape mark and the heel of the lead foot. The participant was instructed to keep this distance and posture in mind when making perceptual judgments later. The participant then completed the step by bringing the trail foot in line with the lead foot and walked back to starting position. Collecting data on a maximum stepping ability prior to perceptual tasks was necessary so that appropriate feedback could be provided to participants in two of the four conditions (see below) and for the sake of experimental control in the other two conditions (see below, cf. Heft, 1993).

In the Focal Condition $(n=20)$, each participant stood with his/her toes just behind the yellow line and closed his/her eyes. The experimenter placed the wooden dowel at one of ten distances from the yellow tape mark $(70-160 \mathrm{~cm}$ in $10-\mathrm{cm}$ increments), and instructed the participant to open his/her eyes. The participant then reported ("yes" or "no") whether it would be possible for him/her to completely step over the dowel using the same criteria as when stepping to a maximum distance. Each participant could take as long as necessary; there was no time limit on a given trial. Once the participant made the "yes" or "no" report, he/she closed his/her eyes while the experimenter placed the dowel at the appropriate distance for the next trial.

In the Timed Condition $(n=20)$, the procedure was identical except that there was a $2 s$ limit on making each perceptual report, which began at the instruction to view the dowel. If the participant did not make the perceptual report within $2 \mathrm{~s}$, the dowel was removed from the mat. After the participant made the "yes" or "no" report, he/she closed his/her eyes while the experimenter placed the dowel at the appropriate distance for the next trial.

In the Puzzle Condition ( $n=18)$, the task of the participant was to complete a "connect-the-dots" puzzle posted on the whiteboard located just behind the participant. The experimenter placed the dowel at the appropriate distance and instructed the participant to open his/her eyes. The participant then reported ("yes" or "no") whether it was possible for him/ her to completely step over the dowel. If the participant was correct (based a comparison of this response with his/her previously recorded maximum distance step), he/she turned around, used a marker to complete the first piece of the puzzle, and then turned back around for the next trial. If the participant was incorrect, the response was recorded, but the participant did not have the chance to complete the piece of the puzzle. As in the Timed condition, there was a 2 -s limit on making each perceptual report, which began at the instruction to view the dowel. If the participant did not make the perceptual report within $2 \mathrm{~s}$, the dowel was removed from the mat. After turning back around or saying "no" the participant closed his/her eyes while the experimenter placed the dowel at the appropriate distance for the next trial. The purpose of the Puzzle 
Condition was to investigate whether nesting the perceptual task within a superordinate task (that did not require stepping or leaping) would influence the accuracy of the perceptual task relative to the Focal Condition (cf. Heft, 1993).

In the Puzzle-Action Condition $(n=20)$, the task of the participant was to complete a "connect-the-dots" puzzle posted on the whiteboard located $340 \mathrm{~cm}$ in front of the participant. The experimenter placed the dowel at the appropriate distance and instructed the participant to open his/her eyes. If the participant felt that it was possible to step over the dowel, he/she did so, walked to the board, used a marker to complete the first piece of the puzzle, and then returned to the starting position. If the participant felt that it was not possible to step over the dowel, he/she said "pass." If the participant attempted but failed to step over the dowel, the trial was recorded as a response of "yes", but the participant did not have the chance to complete the next piece of the puzzle. As in the Timed condition, there was a 2-s limit on initiating the behavior or saying pass, which began at the instruction to view the dowel. If the participant did not make the perceptual report within $2 \mathrm{~s}$, the dowel was removed from the mat. After returning to starting position or saying 'pass' the participant closed his/her eyes while the experimenter placed the dowel at the appropriate distance for the next trial. The purpose of the Puzzle-Action Condition was to investigate whether nesting the perceptual task within a superordinate task (that did require stepping or leaping) would influence the accuracy of the perceptual task relative to the Focal Condition.

In all conditions, the dowel was placed at each distance three times in a random order. Following these 30 trials, the experimenter measured participant weight, standing height, and recorded information about athletic and injury history.

Leaping behavior condition The experimenter measured the participant's maximum leaping distance. The participant was instructed to leap with his/her preferred foot as the lead foot and the non-preferred foot as the trail foot. The participant was instructed that during the leap, the trail foot must leave the ground before the lead foot lands, and that he/she must (eventually) be able to bring the trail foot (and the rest of the body) in line with the lead foot. The experimenter then measured the distance between the tape mark and the heel of the lead foot. The participant was instructed to keep this distance in mind when making perceptual judgments later. The participant then walked back to the starting position.

The Focal $(n=21)$, Timed $(n=19)$, Control $(n=20)$, and Puzzle-Action $(n=20)$ conditions were identical to the respective conditions in Experiment 1 except that perceptual reports and behaviors were with respect to leaping over the dowel as described above.

\section{Results}

Perceived maximum stepping and leaping distances were derived for each participant in each condition. In the Focal, Timed, and Puzzle conditions, this was the longest distance that received a response of "yes" on at least half of the (i.e., on at least two of three) trials in that condition. In the Puzzle-Action Condition, this was the longest distance that the participant attempted to step or leap over on at least half of the (i.e., on at least two of three) trials in that condition.

In what follows, we investigate possible differences in these perceptual boundaries (or measures derived from perceptual boundaries) across behaviors and contexts. To make interpretation of any differences in these measures both more meaningful and more straightforward, we first compared actual stepping/leaping ability for participants in each condition in a 2 (Behavior: Stepping vs. Leaping) $\times 4$ (Context: Focal vs. Timed vs. Puzzle vs. Puzzle-Action) between-participants analysis of variance (ANOVA). There was a main effect of Behavior $F$ (1, $147)=279.19, p<.001, \eta_{\mathrm{p}}{ }^{2}=.66 .-$ actual maximum leaping distance $(M=130.0 \mathrm{~cm}, \mathrm{SD}=17.2 \mathrm{~cm})$ was larger than actual maximum stepping distance $(M=$ $91.1 \mathrm{~cm}, S D=11.6 \mathrm{~cm})$. Importantly, however, neither the main effect of Context nor the Behavior $\times$ Context interaction was significant. In other words, participants assigned to different Context conditions did not have $a$ priori differences in stepping or leaping ability.

\section{Ratio of perceived to actual maximum stepping/leaping distance}

For each participant in the Stepping Behavior group, we divided perceived maximum stepping distance by actual maximum stepping distance. For each participant in the Leaping Behavior group, we divided perceived maximum leaping distance by actual maximum leaping distance. We compared these values in a 2 (Behavior: Stepping vs. Leaping) $\times 4$ (Context: Focal vs. Timed vs. Puzzle vs. Puzzle-Action) ANOVA. There was a significant main effect of Behavior [ $F$ $\left.(1,145)=26.25, p<.001, \eta_{\mathrm{p}}{ }^{2}=.15\right]$ - ratio values were smaller (i.e., closer to 1.0) for Leaping $(M=1.08, S D=0.16)$ than for Stepping $(M=1.22, S D=0.20)$. There was also a main effect of Context $\left[F(3,145)=7.78, p<.001, \eta_{\mathrm{p}}{ }^{2}=.14\right]$. Follow-up t-tests with Bonferroni corrections showed that, overall, ratios were smaller (i.e., closer to 1.0) in the Timed $(M=1.13, S D=0.19)$, Puzzle $(M=1.07, S D=0.10)$, and Puzzle-Action $(M=1.13, S D=0.16)$ conditions than in the Focal Condition $(M=1.25, S D=0.24)$ (all corrected $p$ values $<.05)$ and in no other cases. The interaction of Behavior $\times$ Context was not significant (see Fig. 1). 


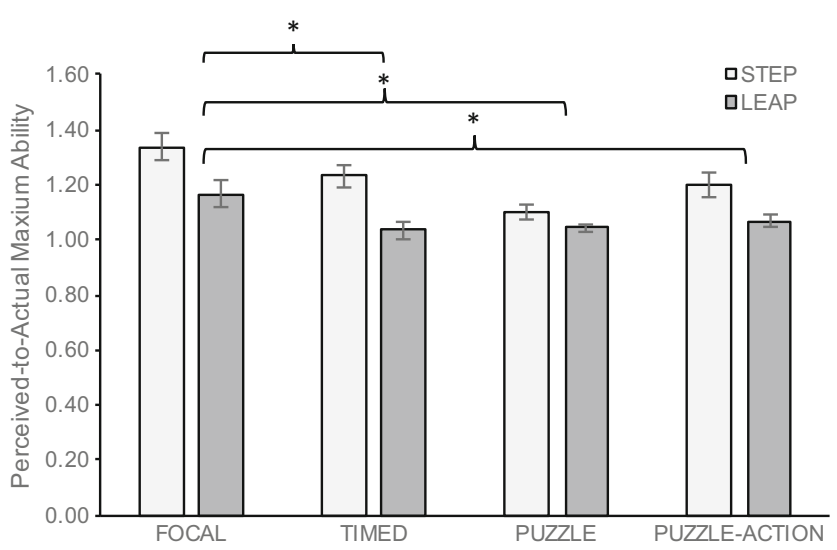

Fig. 1 Perceived-to-actual maximum stepping and leaping distance as a function of Context and Behavior. Error bars indicate standard error. Asterisks $(*)$ indicate significant differences between Context conditions at $p<.05$. There was also a significant main effect of Behavior Condition (Step vs. Leap)

\section{Absolute error}

For each participant in the Stepping Behavior group, we calculated the absolute (i.e., unsigned) error between perceived and actual maximum stepping distance. For each participant in the Leaping Behavior Group, we calculated the absolute (i.e., unsigned) error between perceived and actual maximum leaping distance. We compared these values in a 2 (Behavior: Stepping vs. Leaping) $\times 4$ (Context: Focal vs. Timed vs. Puzzle vs. Puzzle-Action) ANOVA. There was a significant main effect of Behavior $\left[F(1,145)=5.59, p<.05, \eta_{\mathrm{p}}{ }^{2}=.04\right]$ — absolute error was smaller (i.e., showed less overall error) for Leaping $(M=19.5 \mathrm{~cm}, S D=14.7 \mathrm{~cm})$ than for Stepping $(M=14.6 \mathrm{~cm}, S D=12.5 \mathrm{~cm})$. There was also a main effect of Context $\left[F(3,145)=10.94, p<.001, \eta_{\mathrm{p}}{ }^{2}=.19\right]$. Follow-up ttests with Bonferroni corrections showed that, overall, absolute error was smaller in Puzzle $(M=8.6 \mathrm{~cm}, S D=8.1 \mathrm{~cm})$ and Puzzle-Action $(M=15.5 \mathrm{~cm}, S D=10.8 \mathrm{~cm})$ Conditions than in the Focal Condition $(M=24.9 \mathrm{~cm}, S D=16.7 \mathrm{~cm})$ and that absolute error was smaller in the Puzzle-Action than in the Timed condition $(M=18.0 \mathrm{~cm}, S D=13.0 \mathrm{~cm})$ (all corrected $p$ values $<.05)$ and in no other cases. The interaction of Behavior $\times$ Context was not significant (see Fig. 2).

\section{Constant error}

For each participant in the Stepping Behavior group, we calculated the constant (i.e., signed) error between perceived and actual maximum stepping distance. For each participant in the Leaping Behavior group, we calculated the constant (i.e., signed) error between perceived and actual maximum leaping distance. We compared these values in a 2 (Behavior: Stepping vs. Leaping) $\times 4$ (Context: Focal vs. Timed vs. Puzzle vs. Puzzle-Action) ANOVA. There was a significant main effect of Behavior $\left[F(1,145)=16.38, p<.001, \eta_{\mathrm{p}}{ }^{2}=\right.$

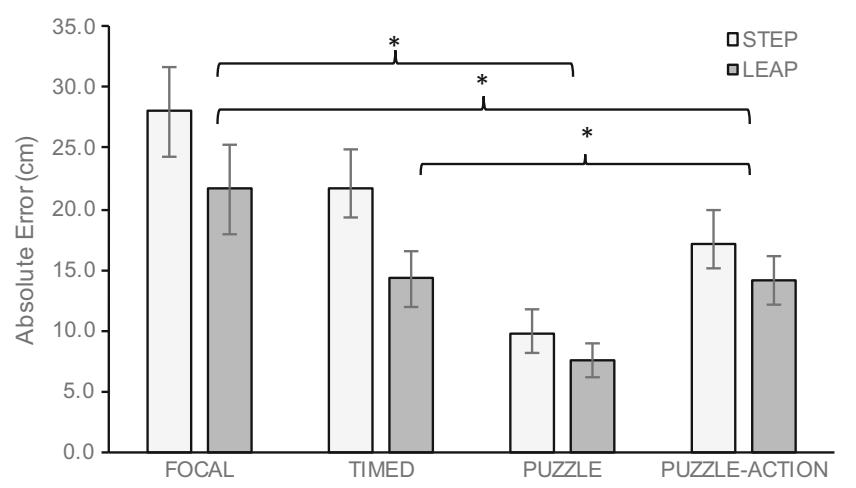

Fig. 2 Absolute (unsigned) error on perceived maximum stepping and leaping distance as a function of Context and Behavior. Error bars indicate standard error. Asterisks $(*)$ indicate significant differences between Context conditions at $p<.05$. There was also a significant main effect of Behavior Condition (Step vs. Leap)

.11] — constant error was smaller (i.e., there was less overestimation) for Leaping $(M=+8.8 \mathrm{~cm}, S D=17.10 \mathrm{~cm})$ than for Stepping $(M=+18.9 \mathrm{~cm}, S D=15.4 \mathrm{~cm})$. There was also a main effect of Context $\left[F(3,145)=6.97, p<.001, \eta_{\mathrm{p}}{ }^{2}=.13\right]$. Follow-up t-tests with Bonferroni corrections showed that, overall, constant error was smaller in the Timed $(M=$ $+12.1 \mathrm{~cm}, \mathrm{SD}=18.6 \mathrm{~cm})$, Puzzle $(M=+7.4 \mathrm{~cm} \mathrm{~cm}, S D=$ $9.2 \mathrm{~cm}$, and Puzzle-Action $(M=+12.2 \mathrm{~cm}, S D=14.5 \mathrm{~cm})$ Conditions than in the Focal Condition $(M=+22.8 \mathrm{~cm}, S D=$ $19.5 \mathrm{~cm}$ ) (all corrected $p$ values $<.05$ ) and in no other cases. The interaction of Behavior $\times$ Context was not significant (see Fig. 3).

\section{Categorization of participants}

Following Heft (1993), we categorized participants in each Behavior and Context Condition into groups based on the magnitude of signed error of perceived maximum stepping or leaping distance (see Tables 1 and 2). A participant was

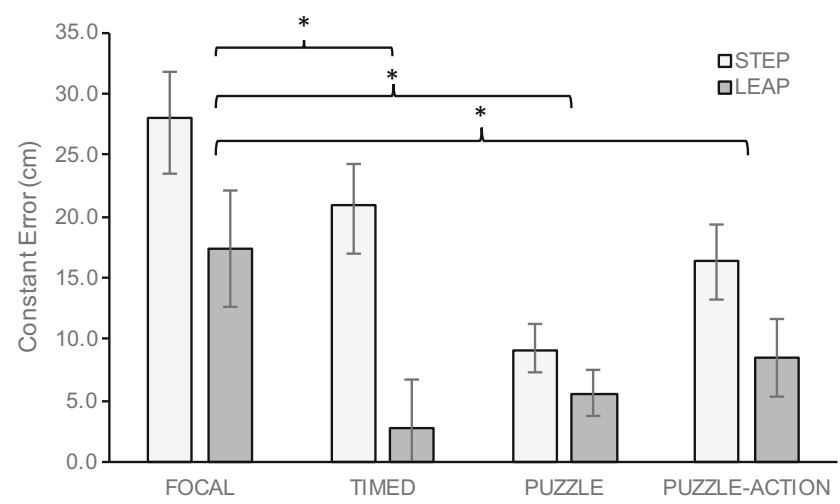

Fig. 3 Constant (signed) error on perceived maximum stepping and leaping distance as a function of Context and Behavior. Error bars indicate standard error. Asterisks $(*)$ indicate significant differences between Context conditions at $p<.05$. There was also a significant main effect of Behavior Condition (Step vs. Leap) 
Table 1 Distribution of participants across context conditions in stepping behavior group

\begin{tabular}{llll}
\hline Conditions & $\begin{array}{l}\text { Underestimate } \\
<-10 \mathrm{~cm}\end{array}$ & $\begin{array}{l}\text { Accurate } \\
-10 \text { to }+10 \mathrm{~cm}\end{array}$ & $\begin{array}{l}\text { Overestimate } \\
>+10 \mathrm{~cm}\end{array}$ \\
\hline Focal $(\mathrm{n}=20)$ & $0(0 \%)$ & $3(15 \%)$ & $17(85 \%)$ \\
Timed $(\mathrm{n}=20)$ & $0(0 \%)$ & $5(25 \%)$ & $15(75 \%)$ \\
Puzzle (n=18) & $0(0 \%)$ & $14(78 \%)$ & $4(22 \%)$ \\
Puzzle-Action $(\mathrm{n}=18)$ & $0(0 \%)$ & $7(39 \%)$ & $11(61 \%)$ \\
\hline
\end{tabular}

categorized as "accurate" if perceived maximum stepping distance was between $-10 \mathrm{~cm}$ and $+10 \mathrm{~cm}$ (i.e., within one placement interval of 0, cf. Heft, 1993). A participant was categorized as "underestimating" if perceived minus actual maximum stepping distance was less (more negative) than $-10 \mathrm{~cm}$; a participant was categorized as "overestimating" if perceived minus maximum stepping distance was greater (more positive) than $+10 \mathrm{~cm}$.

As can be seen in Tables 1 and 2, very few participants were categorized as underestimating in any of the conditions for either Behavior Group (but more so for Leaping Group than for the Stepping Group). In addition, fewer than $20 \%$ of the participants in the Focal Conditions were categorized as accurate in either Behavior Group. By contrast, between $35 \%$ and $80 \%$ of the participants were categorized as accurate in the Timed, Puzzle, and Puzzle-Action conditions were categorized as accurate in each Behavior Group (cf. Heft, 1993).

The distribution of participants for both Behavior Groups combined is shown in Table 3. A chi-square test of independence was performed to examine the relation between Context and accuracy category for the combined dataset. There was a significant relation between these variables $\chi^{2}(6, N=153)=$ $33.92, p<.001$. Post-hoc tests were conducted comparing the obtained and expected distributions of participants within each cell of this matrix (see Beasley \& Schumacker, 1995; Garcíapérez \& Núñez-antón 2003). These Bonferroni corrected tests showed that, in the Puzzle Condition, a disproportionately large number of participants were categorized as Accurate and a disproportionately small number of participants were categorized as Overestimating (in both cases, corrected $p<.05$ ). Conversely in the Focal Condition, a disproportionately small

Table 2 Distribution of participants across context conditions in leaping behavior group

\begin{tabular}{llll}
\hline Conditions & $\begin{array}{l}\text { Underestimate } \\
<-10 \mathrm{~cm}\end{array}$ & $\begin{array}{l}\text { Accurate } \\
-10 \text { to }+10 \mathrm{~cm}\end{array}$ & $\begin{array}{l}\text { Overestimate } \\
>+10 \mathrm{~cm}\end{array}$ \\
\hline Focal $(\mathrm{n}=20)$ & $2(10 \%)$ & $4(19 \%)$ & $14(67 \%)$ \\
Timed $(\mathrm{n}=19)$ & $5(25 \%)$ & $8(40 \%)$ & $6(30 \%)$ \\
Puzzle $(\mathrm{n}=18)$ & $1(5 \%)$ & $14(70 \%)$ & $3(15 \%)$ \\
Puzzle-Action $(\mathrm{n}=20)$ & $2(10 \%)$ & $7(35 \%)$ & $11(55 \%)$ \\
\hline
\end{tabular}

Table 3 Distribution of participants across context conditions with both behavior groups combined

\begin{tabular}{llll}
\hline Context & $\begin{array}{l}\text { Underestimate } \\
<-10 \mathrm{~cm}\end{array}$ & $\begin{array}{l}\text { Accurate } \\
-10 \text { to }+10 \mathrm{~cm}\end{array}$ & $\begin{array}{l}\text { Overestimate } \\
>+10 \mathrm{~cm}\end{array}$ \\
\hline Focal $(\mathrm{n}=40)$ & $2(5 \%)$ & $7(17.5 \%)^{*}$ & $31(77.5 \%)^{* *}$ \\
Time $(\mathrm{n}=40)$ & $5(12.5 \%)$ & $13(32.5 \%)$ & $21(52.5 \%)$ \\
Puzzle $(\mathrm{n}=36)$ & $1(2.8 \%)$ & $28(77.8 \%)^{* *}$ & $7(19.4 \%)^{*}$ \\
Puzzle-Action $(\mathrm{n}=38)$ & $2(5.3 \%)$ & $14(36.8 \%)$ & $22(57.9 \%)$ \\
\hline
\end{tabular}

*Observed value is smaller than expected value at corrected $\mathrm{p}<.05$

$* *$ Observed value is larger than expected value at corrected $\mathrm{p}<.05$

number of participants were categorized as Accurate and a disproportionately large number of participants categorized as Overestimating (in both cases, corrected $p<.05$ ). The latter result is the key finding for our purposes. The fact that a disproportionately small number of participants were classified as Accurate in the Focal Condition (and in no other condition) suggests that, consistent with our hypotheses, perception of maximum stepping and leaping distance was less accurate in this condition than in the other conditions.

Overall, the four analyses show strong support for the first and second hypothesis. Analyses of perceived to actual maximum stepping/leaping distance, absolute error, constant error, and categorization of participants suggest that perception of maximum stepping or leaping distance was more accurate (more closely reflected stepping or leaping ability) when the perceptual task was nested within a superordinate task (the Puzzle and Puzzle-Action Conditions) than when it was not (the Focal Condition). Moreover, for the most part, this difference occurred both when the superordinate task included performing a stepping or leaping task (the Puzzle-Action Condition) and when it did not (the Puzzle Condition). There was also support (though not quite as strong) for the third hypothesis. Analyses of perceived to actual maximum stepping/ leaping distance, constant error, and categorization of participants (but not absolute error) suggested that verbal perceptual reports of maximum stepping or leaping distance were more accurate when there was an explicit time constraint (Timed Condition) than when there was not (Focal Condition).

\section{General discussion}

Many experiments investigating perception of affordances have shown a discrepancy between the perceptual and actual boundaries for a given behavior (e.g., Cole et al., 2013; Higuchi et al., 2004; Wagman \& Morgan, 2010). One possible contributing explanation for such findings is that in such studies, the perceptual task was isolated from the larger context in which it occurred and that this transformed what would have been a perception-action task into an analytical judgment. 
Heft (1993) found support for this hypothesis in his investigation of perception of affordances for reaching. We investigated the same hypothesis with respect to perception of affordances for locomotory behaviors involving the entire body, but that nonetheless are the lower body equivlant of reaching namely stepping and leaping.

In particular, Heft (1993) found that perception of maximum reaching distance more closely corresponded to actual maximum reaching distance when the perceptual task (performing a reach or choosing not to reach) was nested within a superordinate task (completing a puzzle). There were analogous findings in the experiment reported here for perception of maximum stepping and leaping distance. Perception of maximum stepping and leaping distance more closely corresponded to action capabilities for these behaviors when the perceptual task was nested within a superordinate task (completing a puzzle) than when it was not. Moreover, this occurred regardless of whether the superordinate task required performing the behavior (performing a step or leap as in the Puzzle-Action condition) or merely providing a verbal response (as in the Puzzle condition). In other words, for perception of maximum stepping or leaping distance, the nesting of the perceptual task within a superordinate task seemed more critical than the type of response (behavior or verbal judgment) provided in the perceptual task (see Figs. 1, 2, and 3). This is notable given that, at least for some tasks, verbal measures of perception tend to be less reliable, more variable, more malleable, and less accurate than action measures of perception (but see Franchak, Celano, \& Adolph, 2012). It is unclear whether this would also be the case for perception of maximum reaching distance because Heft's experiment did not include a conditon analogous to our Puzzle condition in which providing a verbal response was nested within a superordinate task. This may be a topic of future research.

Heft (1993) also found that perception of maximum reaching distance more closely corresponded to actual maximum reaching distance when there was an explicit time limit on making a verbal perceptual report. For the most part, there were analogous findings for perception of maximum stepping and leaping distance in the experiment reported here. For three of the four analyses, perception of maximum stepping and leaping distance more closely correspended to action capabilities for these behaviors when there was an explicit time constraint on a verbal response (Timed Condition) than when there was not (Focal Condition) (see Figs. 1 and 3 and Tables 1, 2, and 3).

Importantly, the effects of context generalized across perception of maximum stepping and leaping distance. That is, the different contexts did not differentially affect perception of affordances for these behaviors. However, there were differences in perception of affordances for each of these two behaviors. Specifically, analysis of perceived-to-actual maximum stepping/leaping distance, absolute error, constant error, and categorization of participants showed that perception of maximum leaping distance was more accurate (more closely reflected actual ability) than perception of maximum stepping distance (see Figs. 1, 2, and 3). In particular, participants tended to overestimate their stepping ability more so than their leaping ability. This is inconsistent with previous research showing that perceivers tend to underestimate their leaping ability more so than their stepping ability (e.g., Cole et al., 2013; Day et al., 2015). Possible reasons for this inconsistency across studies will be discussed below.

In summary, there were three main findings of the experiment reported here. First, nesting the perceptual task in a superordinate task benefitted the accuracy of both perception of maximum stepping distance and perception of maximum leaping distance. Moreover, the nesting relationship itself seemed more critical than the type of response provided (behavior or verbal report) in the superordinate task. Second, for the most part, providing an explicit time constraint also benefitted the accuracy of both perception of maximum stepping distance and perception maximum leaping distance. Third, in general, perception of maximum leaping distance was more accurate (i.e., more closely correspended to action capabilities) than perception of maximum stepping distance.

\section{Nesting as a contraint on perception and behavior}

The finding that perception of both maximum stepping and leaping distance were each more accurate when the focal (perceptual) task was nested within a superordinate task is consistent with the ecological principle of nesting - the concept that all behaviors (and thus all affordances) are nested in a number of different ways at a number of different scales (Gibson, 1979; Reed, 1996; Stoffregen, 2003a, 2003b; Wagman \& Miller, 2003; Wagman, Caputo \& Stoffregen, 2016). For example, behaviors and affordances are nested within temporal contexts (i.e., one after another) and spatial contexts (i.e., one within another), among others. Nesting a perceptual task within the context of performing a superordinate task respects both this principle and the more general principle that perception is organized with respect to an intended behavioral outcome (Clark, 2015; Gibson, 1979; Noë, 2004). Experimental conditions that do not respect both the nestedness and the goal-orientedness of perception risk transforming a perception-action task into an analytical or reflective judgment and thus altering the nature of the task being investigated (Heft, 1993).

Importantly, the benefits of nesting a focal task within a superordinate task are not only observed in perceptual tasks but also in motor tasks. Previous research has shown that when the attention of the participant was directed toward the movement goal (as opposed to the biomechanical details of the movement itself), movements were both more accurate and more efficient (Wulf, 2007). In such studies, researchers have manipulated the focus of attention by asking the participant to 
direct attention either to specific details of the movements of particular limbs, to the outcomes or effects of those movements, or to a concurrent task such as a memory or communication task (see Keizer, De Bruijn, Smeets, Dijkerman, Postma, 2013; Lopresti-Goodman, Rivera, \& Dressel, 2012).

Focusing attention on the intended outcomes has been found to elicit greater accuracy and efficiency of movement than focusing attention on the movements themselves across a wide range of behaviors including balancing (Wulf, Höss, \& Prinz, 1998, Experiment 2), volleyball serves and soccer passes (Wulf, Gärtner, McConnel, \& Schwarz, 2002), golf chip shots (Wulf \& Su, 2007), basketball free-throws (Zachry, Wulf, Mercer, \& Bezodis, 2005), dart throwing (Marchant, Clough, \& Crawshaw, 2007), and jumping (Becker \& Smith, 2015; Wulf, Zachry, Granados, \& Dufek, 2007).

One interpretation of these effects offered by both Russell (2007) and Oudejans, Koedijker, and Beek (2007) refers to Bernstein's (1967) theory of the control of movement. Specifically, Russell (2007) discussed Bernstein's observation that experienced blacksmiths kept the trajectory of a hammer swung at an anvil relatively invariant, while the trajectories of the joints giving rise to that of the hammer were quite variable. In this sense, the movement is coordinated at the level of the movement's outcome rather than at the level of the joints, muscles, or limbs. From this perspective, focusing on one aspect of a movement may introduce a type of control which is counter-productive to the endpoint control that Bernstein describes. Support for a disruption of movement efficiency when adopting an internal focus has been found by examining joint cross correlations during vertical jumps (Wulf and Dufek, 2009) and soccer kicks (Ford, Hodges, Huys, and Williams, 2009). In both cases, cross correlations among joints increased with an internal focus of attention, suggesting an imposed freezing of degrees of freedom across these joints (Vereijken, van Emmerik, Whiting, \& Newell, 1992), which in turn was associated with reduced maximal jump height and reduced accuracy in soccer kicks. The improved accuracy and efficiency of movement under an external focus of attention may be analogous to the improved accuracy in perception of affordances when the affordance is nested within a superordinate task (and attention is directed toward performing the superordinate task rather than toward the perceptual task itself). In this respect, it could be argued that both perception and behavior are nested in a number of different ways at a number of different scales and are organized with respect to goal to be achieved (Bernstein, 1967; Gibson, 1979).

As described above, in some experimental paradigms in which attention of the participant is manipulated during a perception-action task (including the experiment reported here), attention is typically manipulated by means of nesting the perception-action task within a cognitive task (e.g., a puzzle, memory, or communication task). One disadvantage of doing so is that it may require the participant to switch between performing perception-action and analytic tasks (see Heft, 1993). To this end, it may be instructive to manipulate attention by embedding one perception-action task within another perception-action task (e.g., Wagman et al., 2016; Wagman \& Morgan, 2010). Employing this particular manipulation to the paradigm developed by Heft may also be a topic for future research.

\section{Differences between perception of maximum stepping and leaping distance}

In the current experiment, perception of maximum leaping distance was more accurate (more closely reflected actual ability) than perception of maximum stepping distance. To a large extent, it is not surprising that there are differences in perception of affordances for stepping and for leaping given that there are a number of important differences between these behaviors. Stepping is a non-launching beahvior that is primarily constrained by a static geometric property (i.e., leg length). By contrast, leaping is a (more complex) launching behavior that is additionally constrained by dynamic capabilities (e.g., lower body strength, flexibility, and balance) (see Cole et al., 2013; Day et al., 2015). That is, leaping is necessarily explosive and ballistic; stepping is not. What is somewhat surprising, however, is the direction of the difference in the current experiment. Overall, participants tended to overestimate their stepping ability more so than their leaping ability. In previous research, this difference occurred in the opposite direction - perceivers tended to underestimate their leaping ability more so than their stepping ability (Cole et al., 2013; Day et al., 2015). However, it should be noted that when participants in the current experiment were categorized into groups based on the direction of the error, no participants were categorized as underestimating maximum stepping distance, whereas ten participants (out of 75 or $13 \%$ ) were categorized as underestimating maximum leaping distance.

One possibility for the inconsistency in the direction of the difference is a methodological difference between the experiment reported here and those conducted by Cole et al. (2013) and Day et al. (2015). In the experiment reported here, participants performed a maximum distance step or leap prior to performing the perceptual tasks. As noted above, this was necessary so that appropriate feedback could be provided to participants in the Puzzle and Puzzle-Action conditions and for the sake of experimental control in Focal and Timed Conditions (cf. Heft, 1993). While participants have had a lifetime of experience performing stepping and leaping tasks prior to participating in the experiment, this specific experience of performing a maximum distance step or leap in the context of the experimental procedure may have made the limits of their abilities in these tasks especially salient to them. In turn, this may have influenced perception of maximum stepping distance and, in particular, perception of 
maximum leaping distance. In fact, both Cole et al. (2013) and Day et al. (2015) found that underestimation of perception of maximum leaping distance was reduced following practice performing a leaping task (whereas perception of maximum stepping distance was unchanged following performing a stepping task). It is possible that the context of the experimental task would influence perception of maximum stepping and leaping distance in different ways without this (minimal) experience performing the respective tasks prior to data collection. This may also be a topic of future research.

\section{Addressing potential confounds}

There is at least one additional methodological issue in the experiments reported here that requires attention. In both experiments, participants in the Puzzle and Puzzle-Action Conditions received some form of feedback after each trial in the perceptual task, and participants in the Timed and Focal Conditions did not. Specifically, in the Puzzle condition, the feedback was (explicitly) provided by the experimenter after the participant made his/her verbal report. In the Puzzle-Action condition, feedback was (implicitly) provided when the participant either succeeded or failed at stepping or leaping over the dowel at a given distance. This difference may have contributed to differences in the categorization of participants across these conditions (see Table 3 ). While this is possible, the effect of this difference in whether feedback was provided across conditions is necessarily limited for at least one important reason. In particular, although participants in both the Puzzle and Puzzle-Action Conditions received some form of feedback, participants in these conditions received different types of feedback and different contigencies for feedback. As described above, in the Puzzle-Action Condition, feedback was implicitly provided when the participant either succeeded or failed at performing the behavior, and in the Puzzle Condition, feedback was explicitly provided by experimenter. Moreover, in the Puzzle Condition, the opportunity to complete the next portion of the puzzle was contingent on the accuracy of the verbal response, regardless of whether this response was "yes" or "no." In the Puzzle-Action Condtion, the opportunity to complete the next portion of the puzzle was contingent on successful performance of the stepping or leaping task, but not on accuracy per se. That is, in this condition, the participant had the opportunity to complete the next section of the puzzle only after a successful attempt to step or leap over the dowel; the participant did not have this opportunity after a failed attempt or after a correctly saying "pass." However, for both Behavior Conditions, the Puzzle-Action and the Puzzle Conditions each differed from the Focal Condition, respectively. In other words, feedback (of a particular kind from a particular source) may be sufficient but may not be necessary to benefit the accuracy of perception of maximum stepping or leaping distance. One way to investigate this may be to nest the perceptual task within a superordinate task without providing feedback of any kind (implicit or explicit). This may be another topic for future research.

\section{Conclusion}

A number of studies on perception of affordances have shown a discrepancy between the perceptual boundary for a given behavior and the behavioral boundary for that behavior. One possible theoretically important explanation of such findings is that the conditions of many experimental tasks may not respect the nestedness or goal-directedness of perceptionaction and thus may have transformed dynamic perceptionaction tasks into analytical or reflective judgments. We investigated this hypothesis with respect to perception of maximum stepping and leaping distance. Perception of maximum stepping and leaping distance were each more accurate when the respective perceptual tasks were nested within a superordinate task than when they were not, regardless of whether the behavior itself was performed. In addition, verbal reports of perception of maximum stepping and leaping distance were each more accurate when there was an explicit time limit on perception. The results suggest that nesting a perceptual task within a superordinate task may be necessary to bring perception of affordances in closer correspondence with action capabilities. Under some circumstances performing the behavior, providing verbal reports under an explicit time constraint, or feedback (of a particular kind) may also be sufficient to do so.

Acknowledgments We thank Aja Carten and Daniel Schloesser for their help with data collection.

\section{References}

Adolph, K. E. (2008). Learning to move. Current Directions in Psychological Science, 17, 213-218.

Beasley, T. M., \& Schumacker, R. E. (1995). Multiple regression approach to analyzing contingency tables: Post hoc and planned comparison procedures. The Journal of Experimental Education, 64(1), 79-93.

Becker, K. A., \& Smith, P. J. K. (2015). Attentional focus in standing long jump performance: Influence of a broad and narrow internal focus. Journal of Strength and Conditioning Research, 29, 1780-1783.

Bernstein, N. (1967). The Co-ordination and Regulation of Movements. Oxford: Permagon.

Carello, C., Grosofsky, A., Reichel, F. D., Solomon, H. Y., \& Turvey, M. T. (1989). Visually perceiving what is reachable. Ecological Pychology, 1(1), 27-54.

Clark, A. (2015). Surfing uncertainty. Oxford: Oxford University Press.

Cole, W. G., Chan, G. L. Y., Vereijken, B., \& Adolph, K. E. (2013). Perceiving affordances for different motor skills. Experimental Brain Research, 225, 309-319.

Coren, S. (1993). The lateral preference inventory for measurement of handedness, footedness, eyedness, and earedness: Norms for young adults. Bulletin of the Psychonomic Society, 31, 1-3. 
Day, B. M., Wagman, J. B., \& Smith, P. J. K. (2015). Perception of maximum stepping and leaping distance: Stepping affordances as a special case of leaping affordances. Acta Psychologica, 158, 26-35.

Ford, P., Hodges, N. J., Huys, R., \& Williams, A. M. (2009). Evidence for end-point trajectory planning during a kicking action. Motor Control, 13, 1-24.

Franchak, J. M., \& Adolph, K. E. (2014). Gut estimates: Pregnant women adapt to changing possibilities for squeezing through doorways. Attention, Perception, \& Psychophysics, 76, 460-472.

Franchak, J. M., Celano, E. C., \& Adolph, K. E. (2012). Perception of passage through openings depends on the size of the body in motion. Experimental Brain Research, 223(2), 301-310.

Franchak, J. M., van der Zalm, D. J., \& Adolph, K. E. (2010). Learning by doing: Action performance facilitates affordance perception. Vision Research, 50, 2758-2765.

García-pérez, M. A., \& Núñez-antón, V. (2003). Cellwise residual analysis in two-way contingency tables. Educational and Psychological Measurement, 63(5), 825-839.

Gibson, J. J. (1979). The ecological approach to visual perception. Boston: Houghton Mifflin.

Heft, H. (1993). A methodological note on overestimates of reaching distance: Distinguishing between perceptual and analytical judgments. Ecological Psychology, 5, 255-271.

Higuchi, T., Takada, H., Matsuura, Y., \& Imanaka, K. (2004). Visual estimation of spatial requirements for locomotion in novice wheelchair users. Journal of Experimental Psychology: Applied, 10, 55-66.

Keizer, A., De Bruijn, S. E., Smeets, M. A., Dijkerman, H. C., \& Postma, A. (2013). Walking through apertures: Do you know what you are doing during body-scaled action? Perception, 42(5), 583-585.

Konczak, J., Meeuwsen, H. J., \& Cress, M. E. (1992). Changing affordances in stair climbing: The perception of maximum climbability in young and older adults. Journal of Experimental Psychology: Human Perception and Performance, 18, 691-697.

Lopresti-Goodman, S. M., Rivera, A., \& Dressel, C. (2012). Practicing safe text: the impact of texting on walking behavior. Applied Cognitive Psychology, 26(4), 644-648.

Marchant, D. C., Clough, P. J., \& Crawshaw, M. (2007). The effects of attentional focusing strategies on novice dart throwing performance and their task experiences. International Journal of Sport and Exercise Psychology, 5, 291-303.

Mark, L. S. (1987). Eyeheight-scaled information about affordances: A study of sitting and stair climbing. Journal of Experimental Psychology: Human Perception and Performance, 13, 361-370.

Mark, L. S., Nemeth, K., Gardner, D., Dainoff, M. J., Paasche, J., Duffy, M., \& Grandt, K. (1997). Postural dynamics and the preferred critical boundary for visually guided reaching. Journal of Experimental Psychology: Human Perception and Performance, 23, 1365-1379.

Noë, A. (2004). Action in Perception. Cambridge: The MIT Press.

Oudejans, R. D., Koedijker, J. M., \& Beek, P. J. (2007). An outside view of Wulf's external focus: Three recommendations. E-Journal Bewegung und Training (E-Journal Movement and Training), 1, 41-42.

Reed, E. S. (1996). Encountering the world: Toward an ecological psychology. New York: Oxford University Press.

Regia-Corte, T., \& Wagman, J. B. (2008). Perception of affordances for standing on an inclined surface depends on height of center of mass.
Experimental Brain Research, 191, 25-35. doi:10.1007/s00221008-1492-8

Russell, D. M. (2007). Attentional focus on the invariant control variables. E-Journal Bewegung und Training (E-Journal Movement and Training), 1, 47-48.

Stoffregen, T. A. (2003a). Affordances are enough: Reply to Chemero et al. (2003). Ecological Psychology, 15, 29-36.

Stoffregen, T. A. (2003b). Affordances as properties of the animalenvironment system. Ecological Psychology, 15, 115-134.

Stoffregen, T. A., Yan, C. M., Giveans, M. R., Flanagan, M., \& Bardy, B. G. (2009). Movement in the perception of an affordance for wheelchair locomotion. Ecological Psychology, 21, 1-36.

Vereijken, B., van Emmerik, R. E. A., Whiting, H. T. A., \& Newell, K. M. (1992). Free(z)ing degrees of freedom in skill acquisition. Journal of Motor Behavior, 24, 133-142.

Wagman, J. B., Caputo, S. E., \& Stoffregen, T. A. (2016). Hierarchical nesting of affordances in a tool use task. Journal of Experimental Psychology: Human Perception \& Performance

Wagman, J. B., \& Malek, E. A. (2009). Geometric, kinetic-kinematic, and intentional constraints influence willingness to pass under a barrier. Experimental Psychology, 56, 409-417.

Wagman, J. B., \& Miller, D. B. (2003). Nested reciprocities: The organism-environment system in perception-action and development. Developmental Psychobiology, 42, 317-334.

Wagman, J. B., \& Morgan, L. L. (2010). Nested prospectivity in perception: Perceived maximum reaching height reflects anticipated changes in reaching ability. Psychonomic Bulletin \& Review, 17, 905-909.

Warren, W. H. (1984). Perceiving affordances: Visual guidance of stair climbing. Journal of Experimental Psychology: Human Perception and Performance, 10, 683-703.

Wulf, G. (2007). Attentional focus and motor learning: A review of 10 years of research (Target article). E-Journal Bewegung und Training (E-Journal Movement and Training), 1, 4-14.

Wulf, G., \& Dufek, J. S. (2009). Increased jump height with an external focus due to enhanced lower extremity joint kinetics. Journal of Motor Behavior, 41, 401-409.

Wulf, G., Gärtner, M., McConnel, N., \& Schwarz, A. (2002). Enhancing the learning of sport skills through external-focus feedback. Journal of Motor Behavior, 34(2), 171-182.

Wulf, G., Höss, M., \& Prinz, W. (1998). Instructions for motor learning: Differential effects of internal versus external focus of attention. Journal of Motor Behavior, 30(2), 169-179.

Wulf, G., \& Su, J. (2007). An external focus of attention enhances golf shot accuracy in beginners and experts. Research Quarterly for Exercise and Sport, 78, 384-389.

Wulf, G., Zachry, T., Granados, C., \& Dufek, J. S. (2007). Increases in jump-and-reach height through an external focus of attention. International Journal of Sports Science \& Coaching, 2(3), 275-284.

Yasuda, M., Wagman, J. B., \& Higuchi, T. (2014). Can perception of aperture passability be improved immediately after practice in actual practice? Dissociation between walking and wheelchair use. Experimental Brain Research, 232(3), 753-764.

Zachry, T., Wulf, G., Mercer, J., \& Bezodis, N. (2005). Increased movement accuracy and reduced EMG activity as the result of adopting an external focus of attention. Brain Research Bulletin, 67, 304-309. 